\title{
Southern African Large Telescope spectroscopy of unclassified Fermi-2LAC Active Galactic Nuclei
}

\author{
L. Klindt ${ }^{* \dagger}$ \\ Department of Physics, University of the Free State, Bloemfontein, South Africa \\ E-mail: lizelkeklindtegmail.com \\ B. van Soelen \& P.J. Meintjes \\ Department of Physics, University of the Free State, Bloemfontein, South Africa
}

\section{P. Väisänen \& A. Kniazev}

South African Astronomical Observatory, PO Box 9, 7935, South Africa

Southern African Large Telescope Foundation, PO Box 9, 7935, PO Box 9, 7935, South Africa

\begin{abstract}
We are undertaking a multi-wavelength campaign to classify 19 unclassified Active Galactic Nuclei (AGN) in the 2nd Fermi-LAT catalogue of AGN, which includes optical spectroscopy to establish the targets optical properties and redshifts. With the addition of archival data, we are attempting to establish the Spectral Energy Distributions (SEDs) of these sources, and search for potential Very High Energy (VHE) candidates. The candidate sources all exhibit blazar-like characteristics, are at high galactic latitude $\left(|b|>10^{\circ}\right)$, and the optical/radio counterparts are within the Fermi 95\% error circle. We present optical spectroscopy of 7 (V 20 mag) targets undertaken with the Southern African Large Telescope (SALT) using the Robert Stobie Spectrograph (RSS). The target spectra are mainly featureless as are expected for BL Lac objects. However, we have detected broad emission lines, with equivalent widths greater than $5 \AA$ in the spectra of 2FGL J0044.7-3702 and 2FGL J0201.5-6626, and Ca II K\&H, G-band, MgI and/or NaD absorption lines from the host galaxy are present in 2FGL J0730.6-6607, 2FGL J1154.1-3242 and 2FGL J1218.8-4827, allowing for preliminary redshift measurements of $0.11<z<1.29$. In addition, have obtained a Ca II depression depth value of $\mathrm{K}_{4000}=0.16$. for 2FGL J0730.6-6607, which indicates the contribution from non-thermal jet emission and indicates that the source is of a BL Lac nature.
\end{abstract}

SALT Science Conference 2015 -SSC2015-

1-5 June, 2015

Stellenbosch Institute of Advanced Study, South Africa

\footnotetext{
* Speaker.

${ }^{\dagger}$ This paper uses observations made with the Southern African Large Telescope (SALT).
} 


\section{Introduction}

Jet-dominated Active Galactic Nuclei (AGN), known as blazars, dominate the extragalactic sky and are known to be the most active and violent astronomical objects. Blazars, including BL Lacartae objects (BL Lacs) and flat spectrum radio quasars (FSRQs), exhibit rapid optical variability, high and variable polarization as well as weak or featureless emission line spectra. These extreme properties are believed to be the consequence of relativistically beamed non-thermal emission from the jet which is orientated close to our line of sight (see e.g. [1, 2]). Multi-wavelength analysis provides the opportunity to construct Spectral Energy Distributions (SEDs) of blazar-like sources, which is necessary in order to investigate concepts of galaxy evolution, supermassive black holes (SMBH) and the formation and radiative mechanisms of relativistic jets.

A prominent, well-known feature displayed by blazars is the double-peak structure in the SED. This consists of a low-energy component peaking at radio to UV/X-ray wavelengths and a high-energy component which extends from $\mathrm{X}$-rays to $\mathrm{GeV} / \mathrm{TeV} \gamma$-rays. For the leptonic model, the general interpretation of the mechanisms giving rise to the low-energy and high-energy components are synchrotron and inverse Compton (IC) emission from relativistic electrons, respectively (see e.g. [3]). The IC component is produced either by up-scattering photons from the synchrotron emission within the jet (Synchrotron Self Compton (SSC) scattering), or seed photons originating from the accretion disk around the $\mathrm{SMBH}$, the dust torus or the broad emission line region (External Compton (EC) scattering) (e.g. [4]). The exact mechanisms responsible for the multi-wavelength emission are still under debate and studies have looked at both leptonic and hadronic models as well as searched for correlations/anti-correlations between the different energy bands in order to better constraint the mechanisms (see e.g. [3, 5, 6, 7, 8]).

Different identification schemes have been employed over the years to classify blazars, with specific focus on their optical spectra. BL Lac objects exhibit no or very weak emission lines, and have synchrotron peak frequencies ranging from IR/optical to UV/X-ray energies. Redshift measurements of BL Lacs can be troublesome since the optical continuum is dominated by synchrotron emission emanating from the jet, however, in some cases absorption features, e.g. Ca II H\&K (or $\mathrm{Ca}$ II depression), $\mathrm{Mg}$ I and $\mathrm{NaD}$ lines, produced by the host galaxy's stellar population and/or intervening halos, are present. The detectability of these lines rely on the state and brightness of the central region. Based on the above defining properties, BL Lacs are optically defined as sources with emission and/or absorption lines with equivalent widths ${ }^{1}$ of $\mathrm{W}_{\lambda} \leqslant 5 \AA$, and a diluted Ca break value less than $40 \%$ (see e.g. [9], [10], [11]).

FSRQs comprise of lower synchrotron peak frequencies and have strong narrow and broad emission lines which are produced by thermal disk and broad line region emission. Broad emission lines, such as $\mathrm{H} \beta, \mathrm{Mg}$ II and C IV, in FSRQ spectra can be used to estimate the SMBH mass with the empirical virial scaling relationship [12].

We have searched for candidate blazars among the 157 AGN of unknown type (AGU) sources, with no determined redshifts, listed in the Fermi-LAT 2 year Source catalogue (2LAC) [13]. Our sample comprises of 19 targets of which we place particular focus on 10 targets that are all still identified as AGU in the recently released 3FGL catalogue [14]. The selection criteria we employed are based on those previously employed by [15] in a study of unidentified sources listed in the Third

\footnotetext{
${ }^{1}$ In this paper we will refer to the absolute value of the equivalent width.
} 


\begin{tabular}{lccccccc}
\hline 2LAC Name & Vmag & RA J2000 $_{\text {J }}$ & Dec $_{\text {J2000 }}$ & $\Gamma$ & $S_{4.85}$ & VI & Dates observed \\
\hline J0044.7-3702 & 19.60 & $00: 45: 12$ & $-37: 05: 49$ & 2.57 & 330 & 92.7 & $2015-06-15 \& 2015-07-12$ \\
J0201.5-6626 & 20.56 & $02: 01: 08$ & $-66: 38: 13$ & 2.25 & 168 & 39.8 & $2015-07-23$ \\
J0730.6-6607 & 15.13 & $07: 30: 50$ & $-66: 02: 19$ & 1.34 & 82 & 26.8 & $2014-12-31$ \\
J1106.3-3643 & 17.41 & $11: 06: 24$ & $-36: 46: 59$ & 2.20 & 92 & 24.1 & $2015-03-09$ \\
J1154.1-3242 & 18.88 & $11: 54: 06$ & $-32: 42: 43$ & 2.03 & 212 & 20.5 & $2015-03-09 \& 2015-04-11$ \\
J1218.8-4827 & 17.53 & $12: 19: 02$ & $-48: 26: 28$ & 2.40 & 65 & 26.6 & $2015-03-09$ \\
J1407.5-4257 & 17.47 & $14: 07: 39$ & $-43: 02: 34$ & 1.91 & 149 & 22.9 & $2015-02-09 \& 2015-07-19$ \\
\hline
\end{tabular}

Table 1: AGU candidates in the Fermi-2LAC catalogue with blazar characteristics. The radio counterparts within the $95 \%$ error circle are listed with their corresponding $\mathrm{V}$ magnitude, target position, $\gamma$-ray index $(\Gamma)$, radio flux density at $4.85 \mathrm{GHz}\left(S_{4.85}\right)$, variability index (VI) and the date each target was observed with SALT during Sem I and II 2015.

Energetic Gamma Ray Experiment Telescope catalogue (3EG) [16]. In the Fermi-2LAC catalogue the most likely radio and/or X-ray counterparts to the Fermi sources were established using three different statistical tests which also considered the source density in the region surrounding the Large Area Telescope (LAT) source [13]. For a full discussion of the selection criteria and target list, see [17]. We have carried out a multi-wavelength campaign, including optical spectroscopic and photometric observations, and single dish radio observations, with the aim to classify these sources, and to contribute to our search for Very High Energy (VHE) sources. Here we report on the optical spectroscopic observations of 7 targets listed in our sample, undertaken with the Southern African Large Telescope (SALT) during 2015.

\section{Observations and spectral analysis}

Thus far, spectroscopic observations of 7 AGU targets have been undertaken with SALT during 2014-Semester II and the 2015-Semester I, on the dates summarized in Table 1. We obtained longslit spectra using the Robert Stobie Spectrograph (RSS) [18]. During 2014-Semester II (November 2014 to April 2015) we observed the 5 brighter targets in our sample ( $\mathrm{V}<18 \mathrm{mag}$ ), and during 2015-Semester I (May 2015 to October 2015) we observed 2 fainter targets (V 20 mag) and re-observed 2 targets to increase the signal to noise ratio $(\mathrm{S} / \mathrm{N})$. The different instrument configurations which we utilized for each semester and the data reduction procedure which we followed, are discussed below.

\subsection{4-Semester II}

We used grating pg0300 with two different Camera Stations, $10.0^{\circ}$ (blue configuration) and $11.5^{\circ}$ (red configuration), to obtain a $3200 \AA-9500 \AA$ visible wavelength range with a central resolving power of $R=530$ using a 0.6 " slit. We used 2 spatial dither positions with an offset of 10 arcseconds in order to correct for fringing effects at the longer wavelengths. In total 4 exposures were obtained for the two configurations during grey time for each target.

\subsection{5-Semester I}

The selected targets for this semester were, in general fainter. Therefore, to obtain a higher $\mathrm{S} / \mathrm{N}$, as well as obtain a slightly higher resolution, a different configuration was used. We used 
grating pg0900 with two Camera Stations, $29.5^{\circ}$ (blue configuration) and $38.5^{\circ}$ (red configuration) in order to cover the $4000 \AA-8700 \AA$ visible wavelength range with a central resolving power of $R \sim 860$ using a 2" slit. For the blue configuration we proposed only 1 spatial dither position with two iterations, and for the red configuration we used 2 spatial dither positions with an offset of 10 arcsec in order to correct for fringing effects at the longer wavelengths. Since the exposure times were long ( $\sim 3600 \mathrm{sec}$ per configuration), we separated the blue and red configuration observations to ensure that the total observational time fell within the track length of the source. We also proposed 2 visits for the blue observation in order to stack the data with the aim to increase the S/N ratio.

\subsection{Data reductions}

Data reductions were performed with standard spectroscopic techniques using the IRAF/NOAO packages. For each exposure we performed flat-field correction and cosmic ray rejection. Wavelength calibrations were applied by using an argon arc for the blue configurations and a neon arc for the red configuration, which was taken after each exposure. The exposures were averaged after background correction and then extracted using the NOAO/TWODSPEC package. Each spectrum was flux calibrated with a spectroscopic standard.

The equivalent widths of the broad emission lines were measured utilizing SPECTOOL in IRAF, and the statistical errors in the equivalent widths were determined using the method discussed by [19].

In order to further distinguish between FSRQs and BL Lacs we calculated the Ca II depression using [9]

$$
K_{4000}=\frac{f^{+}-f^{-}}{f^{+}},
$$

where $f^{-}$and $f^{+}$are the average flux values in the between $3750 \AA$ and $3950 \AA$, and $4050 \AA$ and $4250 \AA$ in the rest frame, respectively.

\section{Results and discussion}

The SALT spectra for the 7 targets are shown in Figures 1 to 7 . In our sample we have confirmed the FSRQ nature of 2 sources, as well as the BL Lac nature of 3 sources. The spectroscopic results that we have obtained for each target are discussed below.

2FGL J0044.7-3702: We have obtained a blue and a red configuration spectrum for this source (see Figure 1). A broad emission line is present in the blue spectrum at $5692 \AA$ which is potentially $\operatorname{Mg~II~}\left(\lambda_{\text {rest }}=2798 \AA\right)$. From this the redshift is estimated to be $z \sim 1.03$. We have also estimated to a first approximation the equivalent width of the spectral feature as $\left|\mathrm{W}_{\lambda}\right|=17.9 \pm 3.4 \AA$, from which we speculate that the source is a FSRQ.

2FGL J0201.5-6626: We have obtained the blue configuration spectrum for this source (see Figure 2). Two broad emission line features are detected at $4343 \AA$ and $6398 \AA$, which we have identified as C [III] $\left(\lambda_{\text {rest }}=1909 \AA\right)$ and Mg II $\left(\lambda_{\text {rest }}=2798 \AA\right)$, respectively. The equivalent widths of the 2 lines are $\left|\mathrm{W}_{\lambda}\right|=34 \pm 13 \AA$ and $\left|\mathrm{W}_{\lambda}\right|=42 \pm 12 \AA$, respectively, suggesting the FSRQ nature of the source. We have obtained the redshift measurement as $z \sim 1.29$, which falls within the range of Fermi FSRQs. 
2FGL J0730.6-6607: Absorption features from the host galaxy, i.e. Ca II H\&K ( $\lambda_{H, \text { rest }}=$ $3935 \AA$ and $\left.\lambda_{K, \text { rest }}=3970 \AA\right), \mathrm{Mg} \mathrm{I}\left(\lambda_{\text {rest }}=5174 \AA\right)$ and $\mathrm{NaD}\left(\lambda_{\text {rest }}=5894 \AA\right)$, are present in the spectrum of 2FGL J0730.6-6607, allowing for a redshift estimate of $z \sim 0.11$ (see Figure 3). From Equation 2.1 we have obtained the Ca II depression as $\mathrm{K}_{4000}=0.16$, which suggests that this source is a BL Lac.

2FGL J1106.3-3643: The spectrum is featureless as expected for a BL Lac object (see Figure 4), and therefore we could not determine the redshift for this target. For future studies a higher $\mathrm{S} / \mathrm{N}$ ratio is advised to potentially detect absorption features.

2FGL J1154.1-3242: The Ca II H\&K and G-band ( $\lambda_{\text {rest }}=4306 \AA$ ) absorption lines are potentially present at $4545 \AA, 4593 \AA$ and $5065 \AA$, respectively (see Figure 5). If this is the case, the source has a redshift measurement of $z \sim 1.18$.

2FGL J1218.8-4827: We have measured a redshift of $z \sim 0.15$ from the Ca II H\&K at $4499 \AA$ and $4539 \AA$ absorption lines, as well as the G-band at $4831 \AA$ (see Figure 6).

2FGL J1407.5-4257: The spectrum is mainly featureless as expected for a BL Lac object (see Figure 7).

\section{Conclusion}

The RSS spectra obtained with SALT have yielded promising results in order to classify the Fermi-2LAC AGU targets in our sample. We have detected broad Mg II and/or C [III] emission lines in the spectra of 2FGL J0044.7-3702 and 2FGL J0201.5-6626, with equivalent widths $\left|\mathrm{W}_{\lambda}\right|>5 \AA$ which place these sources in the FSRQ subclass with redshift measurements of $z \sim 1.03$ and $z \sim 1.29$, respectively. For 2FGL J0730.6-6607 we have observed Ca II H\&K, Mg I and $\mathrm{NaD}$ absorption lines with a redshift measurement of $z \sim 0.11$, and a shallow $\mathrm{Ca}$ II depression of $\mathrm{K}_{4000}=0.16$. This places the sources in the BL Lac class. Potential Ca II H\&K and G-band absorption lines from the host galaxy are present in the spectra of 2FGL J1154.1-3242 and 2FGL J1218.8-4827, which allows for a first approximation of the redshift measurements as $z \sim 0.18$ and $z \sim 0.15$, respectively. The remaining 2 targets, for which spectroscopic observations have also been undertaken, exhibit featureless spectra, and therefore, it may be interpreted that the targets are also potentially BL Lac objects. However, further observations and analysis are required.

\section{Acknowledgments}

The financial assistance of the National Research Foundation (NRF) towards this research is hereby acknowledged. This work is based on the research supported in part by the National Research Foundation of South Africa for the grant 87919. Any opinion, finding and conclusion or recommendation expressed in this material is that of the authors and the NRF does not accept any liability in this regard. This paper uses observations made with the Southern African Large Telescope (SALT).

\section{References}

[1] A.A. Abdo et al., Spectral Properties of Bright Fermi-detected Blazars in the Gamma-ray Band, ApJS 188 (2010) 405 
[2] P.L. Nolan et al., Fermi Large Area Telescope Second Source Catalog, ApJS 199 (2012) 31

[3] M. Böttcher, A. Reimer, K. Sweeney and A. Prakash, Leptonic and Hadronic Modeling of Fermi-detected blazars, ApJ 768 (2013) 54

[4] A.E. Wehrle et al., Multiwavelength Observations of a Dramatic High-Energy Flare in the Blazar 3C 279, ApJ 497 (1998) 178

[5] K. Mannheim and P.L. Biermann, Gamma-ray flaring of 3C 279: a proton initiated cascade in the jet?, $A \& A 253$ (1992) L21

[6] F.A. Aharonian, TeV Gamma Rays from BL Lac Objects due to Synchrotron Radiation of Extremely High Energy Protons, NewA, 5 (2000) 377-395

[7] A. Mücke and R.J. Protheroe, A proton synchrotron blazar model for flaring in Markarian 501, APh 15 (2001) 121

[8] A. Mücke, R.J. Protheroe, R. Engel, J.P Rachen and T. Stanev, BL Lac objects in the synchrotron proton blazar model, APh 18 (2003) 593

[9] M.J.M. Marchã, I.W.A. Browne, C.D. Impey and P.S. Smith, Optical spectroscopy and polarization of a new sample of optically bright flat radio spectrum sources, MNRAS 281 (1996) 425

[10] H. Landt, P. Padovani and P. Giommi, The classification of BL Lacertae objects: the Ca H\&K break, MNRAS 336 (2002) 945-956

[11] E. Galbiati et al., XMM-Newton spectroscopy of an X-ray selected sample of RL AGNs, A\&A 430 (2005) 927

[12] R.J. McLure and J.S. Dunlop, Measuring the black hole masses of high-redshift quasars, MNRAS 337 (2002) 109

[13] M. Ackermann et al, The second catalog of Active Galactic Nuclei detected by the Fermi Large Area Telescope, ApJ 171 (2011) 37

[14] F. Acero et al., Fermi Large Area Telescope Third Source Catalog, ApJ (2015) arXiv:1501.02003

[15] P. Nkundabakura and P.J. Meintjes, Unveiling the nature of two unidentified EGRET blazar candidates through spectroscopic observations, MNRAS 427 (2012) 859-871

[16] R.C. Hartman et al., The Third EGRET Catalog of High-Energy Gamma-Ray Sources, ApJS 123 (1999) 79-202

[17] L.Klindt, P.J. Meintjes and B. van Soelen, Multi-wavelength classification of unidentified AGN in the Fermi-2LAC catalogue, Proceedings of SAIP (2014) 341-346

[18] E.B. Burgh et al., Prime Focus Imaging Spectrograph for the Southern African Large Telescope: optical design, SPIE 4841 (2003) 1463

[19] K. Vollmann and T. Eversberg, Remarks on statistical errors in equivalent widths, Astronomische Nachrichten 327 (2006) 862 

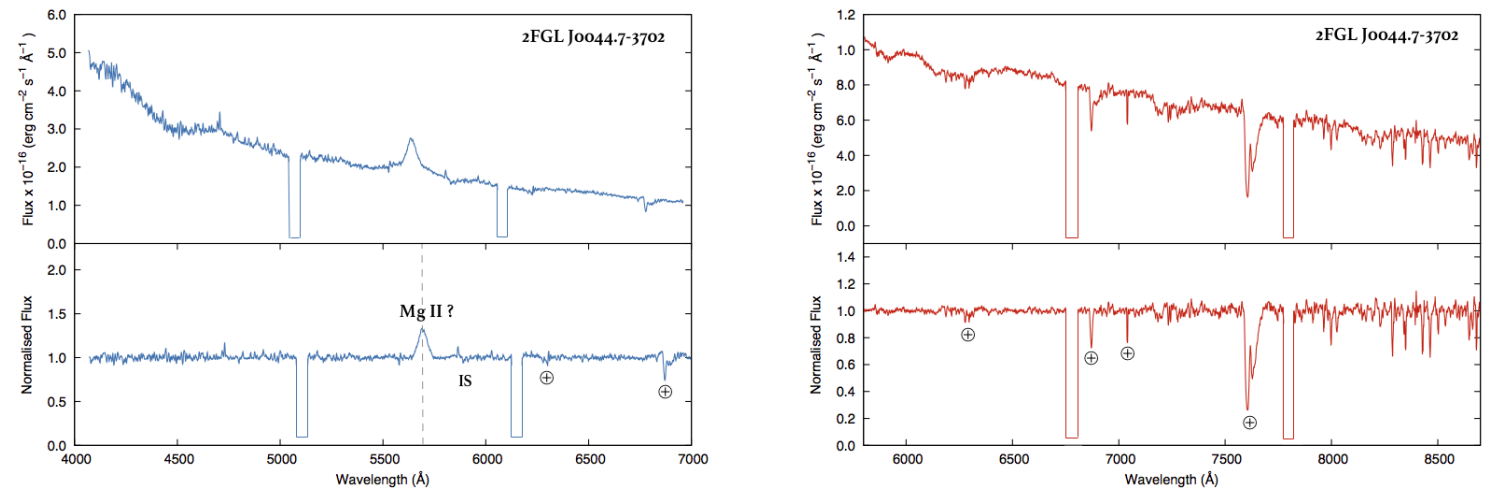

Figure 1: Top panels: optical spectra of 2FGL J0044.7-3702, potentially classified as a FSRQ at $z \sim 1.03$ based on the Mg II emission line. Bottom panels: normalized spectra. Telluric lines are indicated with circles and absorption features due to the interstellar medium of our galaxy with IS. The spectra recorded with the blue and red configurations are shown on the left and right, respectively.

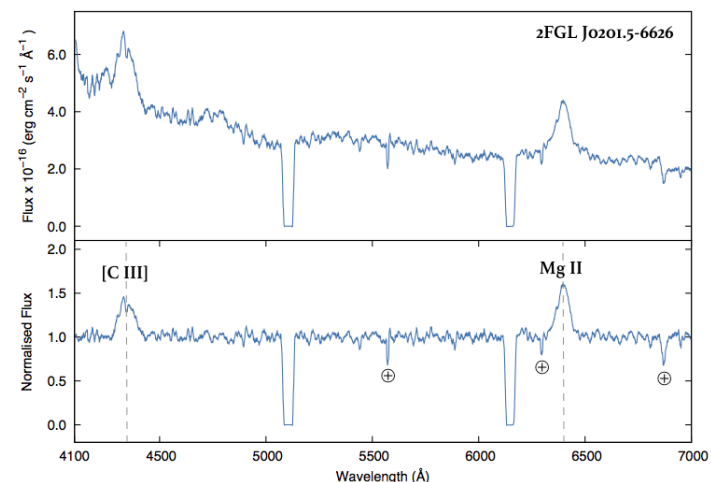

Figure 2: Top panel: optical spectrum of 2FGL J0201.56626 , potentially classified as a FSRQ at $z \sim 1.29$ based on the $\mathrm{C}$ [III] and Mg II emission lines. Bottom panel: normalized spectrum. Telluric lines are indicated with circles and absorption features due to the interstellar medium of our galaxy with IS.

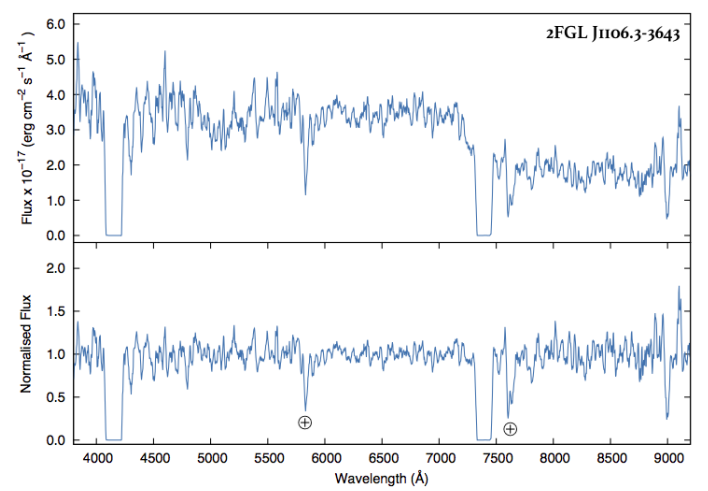

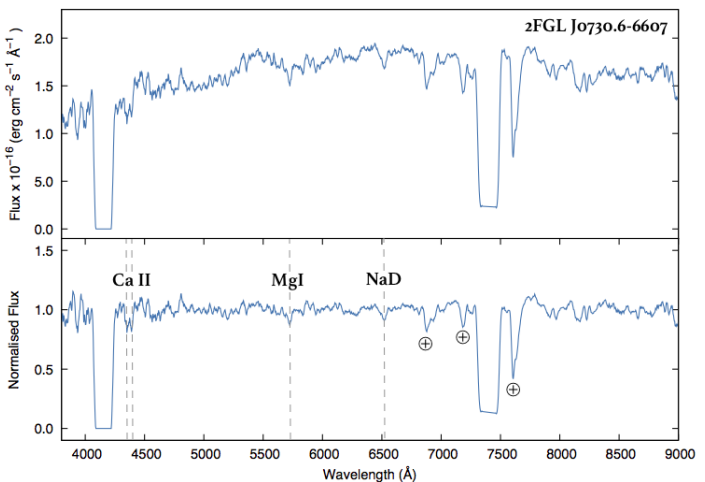

Figure 3: Top panel: optical spectrum of 2FGL J0730.66607, potentially classified as a BL Lac object at $z \sim 0.11$ based on the $\mathrm{Ca}$ II $\mathrm{H} \& \mathrm{~K}, \mathrm{Mg} \mathrm{I}$ and $\mathrm{NaD}$ absorption lines. Bottom panel: normalized spectrum. Telluric lines are indicated with circles.

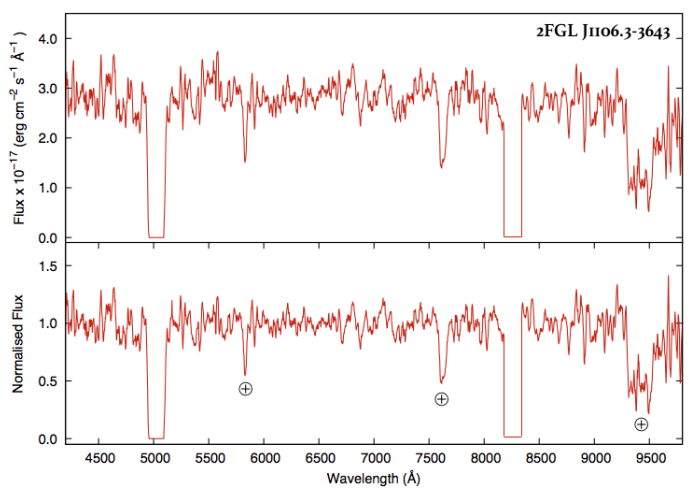

Figure 4: Top panels: optical spectra of 2FGL J1106.3-3643. The spectra lack emission and absorption features and therefore, no redshift estimate could be made. From this we deduce that the source is potentially a BL Lac object. Bottom panels: normalized spectra. Telluric lines are indicated with circles. The spectra recorded with the blue and red configurations are shown on the left and right, respectively. 


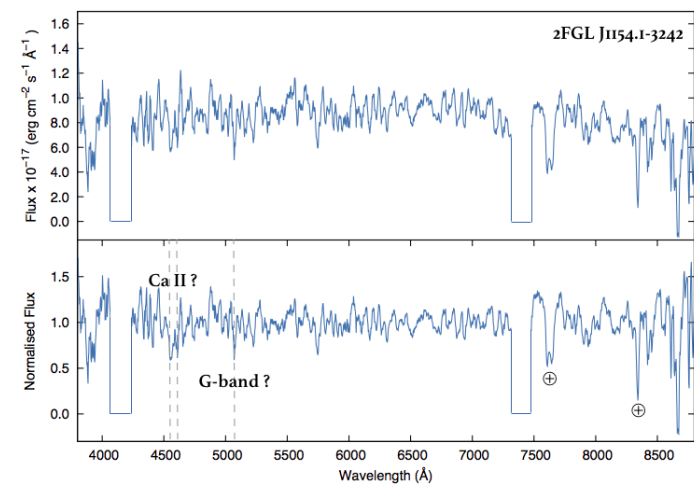

Figure 5: Top panel: optical spectrum of 2FGL J1154.13242, potentially classified as a BL Lac object at $z \sim 0.18$ based on the Ca II H\&K and G-band absorption lines. Bottom panel: normalized spectrum. Telluric lines are indicated with circles and absorption features due to the interstellar medium of our galaxy with IS.

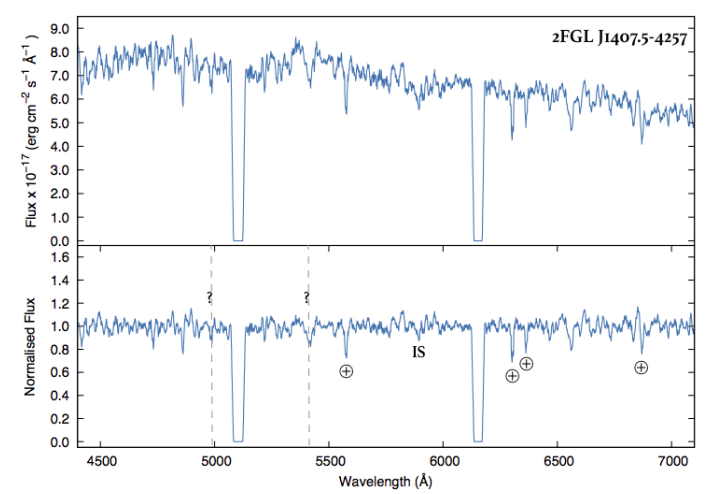

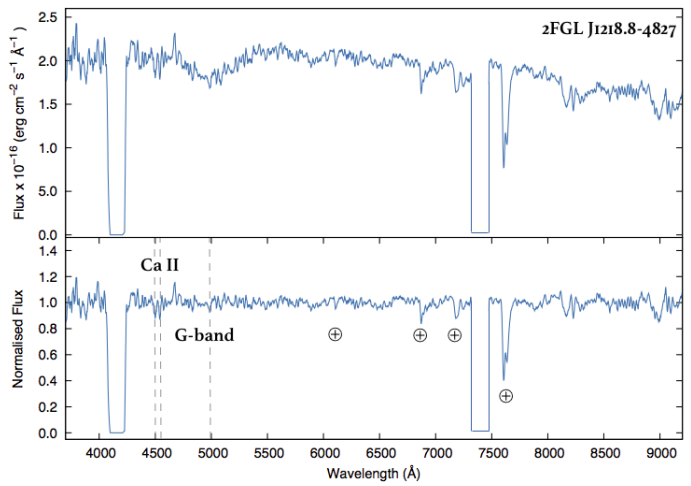

Figure 6: Top panel: optical spectrum of 2FGL J1218.84827, potentially classified as a BL Lac object at $z \sim 0.16$ based on the Ca II H\&K and G-band absorption lines. Bottom panel: normalized spectrum. Telluric lines are indicated with circles.

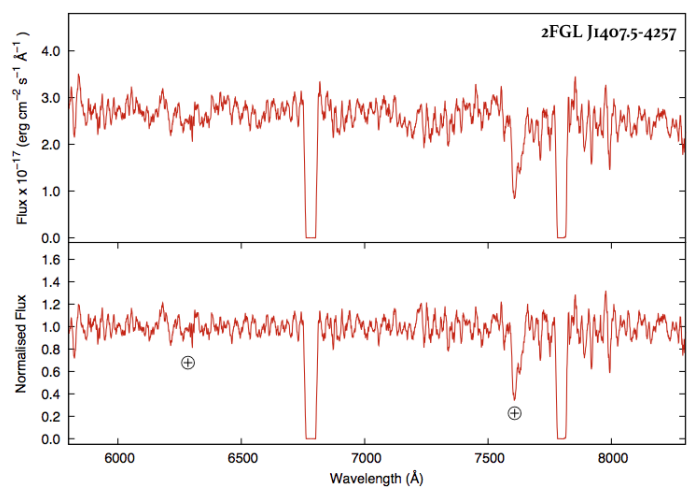

Figure 7: Top panels: optical spectra of 2FGL J1407.5-4257. The spectra lack emission lines, with potential absorption lines at $4984 \AA$ and $5409 \AA$, and therefore, show potential BL Lac nature. Bottom panels: normalized spectra. Telluric lines are indicated with circles and absorption features due to the interstellar medium of our galaxy with IS. The spectra recorded with the blue and red configurations are shown on the left and right, respectively. 